\title{
Qualidade de vida e satisfação no trabalho de funcionários técnico administrativos da Universidade Federal de Santa Maria
}

\author{
Ana Paula Ziegler Vey ${ }^{1}$, Luciane Sanchotene Etchepare Daronco ${ }^{2}$, Andressa Ferreira da Silva ${ }^{3}$, \\ Leonardo Fernandes de Souza ${ }^{3}$, Melissa Medeiros Braz ${ }^{4}$, Haury Haury Temp ${ }^{5}$, \\ Nadyne Nadyne Rubin ${ }^{3}$, Tábada Samantha Marques Rosa ${ }^{6}$
}

\begin{abstract}
RESUMO
O objetivo deste estudo foi avaliar a qualidade de vida dos funcionários da Universidade Federal de Santa Maria e a satisfação no trabalho dos mesmos. A amostra foi composta por 35 funcionários técnico administrativos, de ambos os sexos, que trabalhavam na Universidade Federal de Santa Maria, que atenderam ao chamado do estudo e aceitaram participar. Para avaliação utilizou-se uma ficha de avaliação adaptada de Queiroga, o questionário de qualidade de vida SF36, o teste das figuras de Stunkard e por fim o índice de satisfação do trabalho. Encontrou-se uma idade média de $42 \pm 13,2$ anos, o escore de qualidade de vida encontrou-se satisfatório bem como a satisfação no trabalho, porém o estudo demonstrou que os indivíduos apresentaram insatisfação com relação à imagem corporal.
\end{abstract}

Descritores: Qualidade de Vida; Recursos Humanos; Fisioterapia.

\section{Quality of life and job satisfaction of administrative technician employers of University Federal of Santa Maria}

\begin{abstract}
The objective of this study was to evaluate the quality of life of employees of the Federal University of Santa Maria and their job satisfaction. The sample consisted of 35 administrative technical staff, of both sexes, who worked at the Federal University of Santa Maria, which responded to the call of the study and agreed to participate. For evaluation used an evaluation form adapted Queiroga, the questionnaire of quality of life SF36, the test of Stunkard figures and finally the job satisfaction index. We found a mean age of $42 \pm 13.2$ years, scores of quality of life was found satisfactory and job satisfaction, but the study showed that the participants had dissatisfaction with body image.
\end{abstract}

Descriptors: Quality of Life; Human Resources; Physical Therapy.

\footnotetext{
${ }^{1}$ Especialista em Traumato-Ortopédica pela Universidade Ingá (UNINGÁ), Santa Maria, RS, Brasil.

${ }^{2}$ Doutora em Ciência do Movimento Humano pela Universidade Federal de Santa Maria (UFSM), Santa Maria, RS, Brasil.

${ }^{3}$ Especialista em Atividade Física, Desempenho Motor e Saúde pela Universidade Federal de Santa Maria (UFSM), Santa Maria, RS, Brasil.

${ }^{4}$ Doutora em Engenharia de Produção pela Universidade Federal de Santa Catarina (UFSC), Florianópolis, SC, Brasil.

${ }^{5}$ Especialista em Ciência do Movimento Humano pela Universidade Federal de Santa Maria (UFSM), Santa Maria, RS, Brasil.

${ }^{6}$ Mestre em Distúrbios da Comunicação Humana pela Universidade Federal de Santa Maria (UFSM), Santa Maria, RS, Brasil.
} 


\section{Introdução}

É inegável que, no curso da história, o trabalho de uma forma geral foi humanizado. A jornada de trabalho diminuiu, as condições de trabalho melhoraram, o ser humano ganhou centralidade, mas com isto vieram algumas mudanças no que se refere ao modo de execução do trabalho e a exigência física e psicológica do trabalhador ${ }^{1}$.

Após o período da Revolução Industrial a inserção da tecnologia no ambiente de trabalho foi inevitável, principalmente a do computador. Esta nova fase fez aumentar a carga de trabalho (maior número de tarefas a serem executadas no mesmo espaço de tempo) e deixou o ambiente de trabalho mais individualizado (devido à diminuição dos contatos humanos)2.

A ocupação com a tecnologia, com ambientes individualizados e com menos esforço físico, forçou os funcionários a trabalharem na postura sentada, que gera, por sua vez, posturas paradoxais: enquanto segmentos corporais permanecem estáticos por longos períodos de tempo, como a coluna vertebral, outros como os membros superiores (braços) precisam realizar movimentos altamente repetitivos, que predispõe ao aparecimento de lesões. Nesse sentido, além do desgaste físico do trabalho estático e especializado, há também a necessidade de concentração em sua execução (por mais simples que o trabalho seja) ) $^{3,4}$.

Todos esses fatores podem causar danos à saúde do trabalhador, que passa de peça chave da empresa para um mero prestador de serviço, ficando ele a mercê das doenças ocupacionais ${ }^{5}$. A evidência das doenças ocupacionais no ambiente de trabalho é clara, como também a diminuição da produtividade e perda progressiva na qualidade vida ${ }^{6,7}$.

A qualidade de vida consiste na busca de situações prazerosas onde ter saúde significa usufruir o bem estar propiciado pelo nível de capacidade física, emocional, intelectual e social; ${ }^{5}$ este termo vem ganhando espaço no meio acadêmico e também fora dele. Um exemplo é a adoção de programas que visem à promoção da saúde e qualidade de vida dos funcionários, por parte das empresas ${ }^{6,8,9}$. A pesquisa sobre saúde do trabalhador pode ser considerada uma ramificação do estudo da qualidade de vida geral e vem recebendo atenção crescente de pesquisadores das áreas de saúde ${ }^{10,11,}$ buscando melhoria nas condições de trabalho e de vida destes. Logo, é importante realizar uma boa avaliação da qualidade de vida do trabalhador para que tanto a empresa quanto o mundo acadêmico possam entender a satisfação do empregado como os valores, atitudes e objetivos pessoais que precisam ser identificados, compreendidos e estimulados para que estes consigam sua efetiva participação na empresa, devendo esta conter aspectos como performance no trabalho, e as funções de saúde e relações sociais enfatizando a experiência subjetiva de vida do paciente ${ }^{12,13}$. Além disso, a satisfação no trabalho pode refletir em vários pontos da organização. Um impacto na empresa pode vir da relação da satisfação com o desempenho do empregado, fazendo-se necessário seu estudo.

Dentro das áreas da saúde destaca-se a fisioterapia do trabalho, que é uma especialidade surgida diante da necessidade do acompanhamento da saúde do trabalhador em geral e buscando sua qualidade de vida, baseada em algumas ciências, dentre elas a ergonomia, a biomecânica e atividade física laboral, atuando na avaliação, prevenção, resgate e manutenção da saúde do trabalhador ${ }^{14}$. Portanto, o objetivo deste estudo foi avaliar a qualidade de vida e satisfação no trabalho de funcionários técnico administrativos da Universidade Federal de Santa Maria.

\section{Metodologia}

Conforme a resolução 466/2012 do Conselho Nacional de Saúde, do Ministério da Saúde (CNS/MS) sobre Pesquisas com Seres Humanos (BRASIL, 1996), este estudo recebeu Aprovação do Comitê de Ética e Pesquisa da UFSM antes do início da coleta de dados em 09 de dezembro de 2012, protocolo número 07564112.5.0000.5346.

Tratou-se de uma pesquisa correlacional e de cunho quantitativo ${ }^{15,16}$. Fizeram parte da amostra 35 funcionários técnico administrativos da Universidade Federal de Santa Maria, que leram e assinaram o TCLE, sendo destes 17 do sexo feminino e 18 do sexo masculino. Destes, 18 não tinham filhos, 33 não eram tabagistas, a idade média foi de 42 $\pm 13,2$ anos.

Os servidores administrativos atuam em diversos processos nas instituições de ensino superior e são responsáveis, dentre outras atividades, pelo trâmite de documentações de diversas naturezas, estando submetidos ao cumprimento de prazos e metas, além da esperada qualidade dos serviços a realizar. ${ }^{17}$

Considerou-se considerados critérios de inclusão: funcionários técnico administrativos da UFSM que trabalham em regime de dedicação exclusiva, por não apresentarem acúmulo de funções, o que poderia ser um viés na interpretação dos resultados e como critérios de exclusão do estudo o pesquisado apresentar déficit cognitivo (não podendo assim 
entender e compreender os questionários propostos), não trabalhar em regime de dedicação exclusiva e o funcionário que não apresentasse vínculo empregatício vitalício com a UFSM.

O convite para a pesquisa realizou-se via site, via rádio da própria universidade e por convite direto nos centros da Universidade, não foi realizado cálculo amostral pois almejava-se neste estudo avaliar todos (100\%) os funcionários que ouvissem o chamado da pesquisa e que se encaixassem nos critérios de inclusão e exclusão. Os funcionários que entraram em contato receberam instruções sobre o estudo bem como leram e assinaram o Termo de Consentimento Livre e Esclarecido (TCLE) e após isso foram acolhidos e avaliados.

A coleta de dados realizou-se nos meses de fevereiro, março e junho de 2013 na sala 3004 do Centro de Educação Física e Desportos (CEFD) do campus da UFSM, prédio 51 e também na própria sala do funcionário se assim este preferisse. As coletas ocorreram de forma padronizada e individual (pesquisador-pesquisado).

Utilizou-se para coleta de dados:

[1] Ficha de avaliação adaptada de Queiroga ${ }^{17}$, composta pelos dados de identificação, sócio-demográficos de cada participante, dados sobre as condições de trabalho, situação laboral e dor músculo esquelética (Escala Análogo Visual (EVA) e diagrama corporal) ${ }^{18}$.

[2] Questionário SF 36, este questionário é multidimensional, formado por 36 itens, englobados em oito domínios podendo ser agregados em dois grandes subgrupos: 1 - Físico englobando capacidade funcional, aspectos físicos, dor e estado geral de saúde e 2 - Mental referindo-se à saúde mental, aspectos emocionais, aspectos sociais e vitalidade. Neste contexto 0 questionário SF 36 abrange grande parte destes aspectos subdividindo em oito domínios a qualidade de vida: capacidade funcional, dor corporal, vitalidade, saúde geral, função social, função física e emocional e saúde mental. Apresenta um escore final de 0 a 100, no qual zero corresponde ao pior estado geral de saúde e 100 ao melhor estado de saúde ${ }^{19}$;

Como a qualidade de vida abrange outros quesitos utilizou-se também

[3] Índice de satisfação no trabalho (OSI): traduzido e validado para o português por Swan et al (1993) ${ }^{20}$, este instrumento possibilita a mensuração da satisfação com 22 aspectos psicossociais no trabalho por meio de escalas de Likert de seis pontos, indo de "enorme insatisfação" a "enorme satisfação". A soma dessas medidas fornece um indicador global de satisfação no trabalho, dado por um escore, que varia de 22 a 132 pontos. Para avaliação da satisfação em cada aspecto do trabalho, as variáveis serão categorizadas em: insatisfação (enorme insatisfação e muita insatisfação), satisfação intermediária (alguma insatisfação e alguma satisfação) e satisfação (muita satisfação e enorme satisfação). ${ }^{20}$

[4] Teste das figuras de Stunkard: apresenta nove silhuetas femininas e nove masculinas, cada uma com um número correspondente, organizadas em tamanho crescente da esquerda para direita, desde um corpo muito magro, que seria 0 número um, até a representação de um corpo com obesidade, que corresponderia ao número nove. 0 pesquisado deverá escolher figura que mais parece com o seu corpo e a figura que ele gostaria de se parecer ${ }^{21}$. As figuras foram apresentadas aos funcionários, e, em seguida, eles responderão as seguintes perguntas: 1. Qual a silhueta que melhor representa a sua aparência física atual (Silhueta real)? 2. Qual a silhueta que você gostaria de ter (Silhueta ideal)? A insatisfação com a imagem corporal será identificada por meio da diferença entre o valor da silhueta real e o valor indicado como a silhueta ideal (SILHUETA REAL - SILHUETA IDEAL). Os funcionários que apresentassem valor igual a zero foram classificados como "Satisfeitos" com a imagem corporal, e aqueles que apresentassem valores diferentes de zero como "Insatisfeitos". Quando essa diferença for positiva, o indivíduo será considerado insatisfeito por excesso de peso, e quando negativa, como insatisfeito por magreza.

Ao final da pesquisa, desenvolveu-se uma cartilha com os resultados deste estudo e orientações de cuidados em relação à postura no trabalho para os funcionários. A cartilha foi entregue individualmente, no local de trabalho. As pesquisadoras também se colocaram à disposição para dirimir possíveis dúvidas.

Os dados foram armazenados em planilha eletrônica do tipo Excel 2010. Inicialmente foi realizada a análise descritiva das variáveis. Para a avaliação da normalidade das variáveis foi utilizado o teste de Shapiro-Wilk. Na análise inferencial foram utilizados os testes não paramétricos: Qui-Quadrado, U de Mann-Whitney e Correlação de Spearman. Foi considerado o nível de significância de 10\%. As análises foram realizadas utilizando-se o aplicativo computacional STATISTICA 9.1.

\section{Resultados}

A análise da ficha de avaliação com relação à situação laboral atual permitiu identificar que a média de trabalho em anos na empresa é de 13,1 $\pm 12,8$ anos, sendo que 26 indivíduos desenvolvem suas atividades laborais predominantemente na posição sentada, já 1 predominantemente na posição em pé e 8 em ambas as posições. 
Ainda quanto à situação laboral, quando indagados sobre o cansaço produzido pela função, 27 referiram apresentar o quadro sugerido e quanto à natureza do cansaço, 9 manifestaram cansaço mental, 2 somente cansaço físico e 16 manifestaram ambos (físico e mental).

Já com relação à demanda exigida pela função, 21 consideraram a demanda mental, 7 apresentaram somente física, e outros 7 se mostraram com ambas resultantes da demanda de trabalho.

Quando questionados acerca da dor músculo esquelética 21 voluntários responderam afirmativamente embora tivessem a opção de pontuar mais de um local doloroso e 14 não apresentaram dor. Sendo que os pontos mais referidos foram coluna cervical (8), ombros (6), coluna torácica (7) e coluna lombar (9).

Já com relação ao índice de satisfação no trabalho, os escores variaram de 22 a 132, com média de $96 \pm 16$ pontos, 0 que indica que os funcionários apresentam-se satisfeitos com sua atividade laboral.

Os resultados do questionário SF36 estão apresentados na figura 1, sugerindo que a qualidade de vida está satisfatória, sendo que os quesitos mais elevados foram a capacidade funcional (84 pontos) e os aspectos físicos (84 pontos) e os mais prejudicados foram vitalidade (61 pontos), dor (68 pontos), saúde mental (69 pontos) e aspectos gerais de saúde (69 pontos).

Quando questionado aos voluntários se estavam satisfeitos com sua massa corporal 23 indivíduos relataram insatisfação, sendo que 22 destes gostariam de diminuir seu peso, dado confirmado no teste das figuras de Stunkard, onde foi constatado que na imagem real do indivíduo a média da população estudada optou pela silhueta 5 e quando inquiridos qual a imagem ideal a média da população estudada respondeu a silhueta 3, como mostrado na figura 1.

Figura 1: Escores dos domínios do questionário de qualidade de vida em saúde SF-36 dos técnicos administrativos.

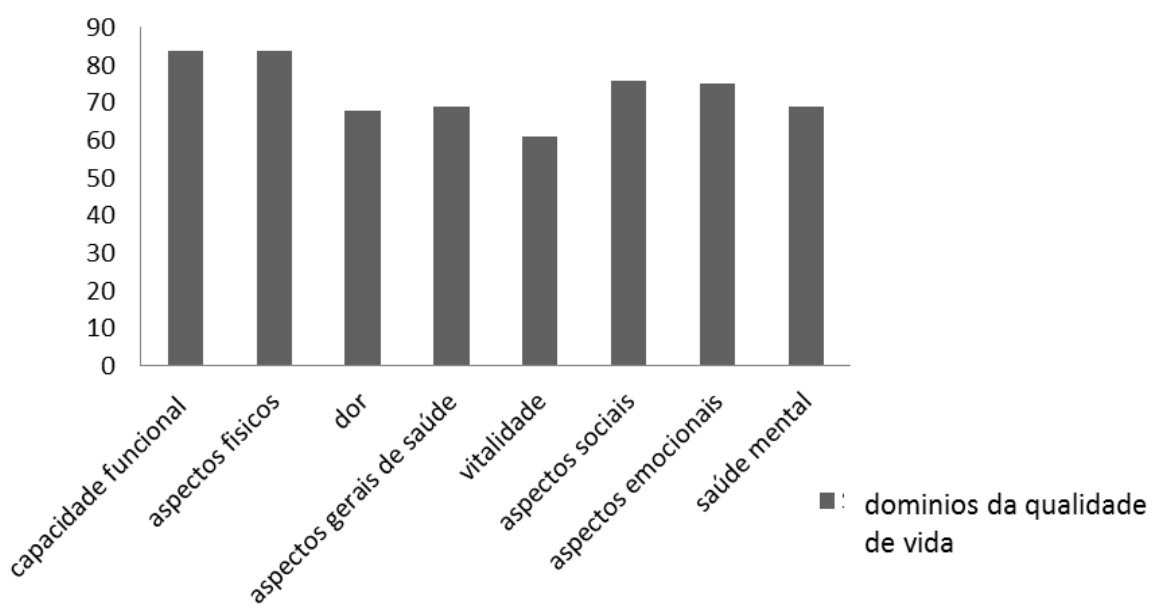

$\mathrm{Na}$ figura 2, os círculos em volta das figuras representam: círculo tracejado representa a imagem ideal, círculo com pontilhados representa a imagem real.

A única correlação significativa entre os domínios do SF-36 e o IST ( $p$-valor=0,084) foi entre o escore de aspecto físico e a satisfação no trabalho. Quanto maior o escore do aspecto físico maior o valor do IST, ou seja, houve uma correlação positiva $(r=0,295)$. Todas as outras correlações não foram significativas.

Foi avaliada a correlação entre os domínios do SF-36 e o teste das figuras, sendo que houve correlação significativa ( $p$-valor $=0,064$ ) entre o escore de aspecto físico e a diferença entre a imagem ideal e a real (teste de figuras). Quanto maior o escore do aspecto físico menor a diferença, ou seja, houve uma correlação negativa ( $r=-0,316)$.

Também houve correlação significativa ( $p$-valor=0,072) entre o escore de aspectos sociais e a diferença entre a imagem ideal e a real (teste de figuras). Quanto maior o escore dos aspectos sociais menor a diferença, ou seja, houve uma correlação negativa ( $\mathrm{r}=-0,307)$.

Sobre a prática de atividade física, não houve associação significativa ( $p$ valor $=0,129$ ) entre a prática de atividade física e a presença de dor muscular.

Quando comparado o tempo de trabalho dos que apresentavam dor musculoesquelética com os que não apresentavam, constatou-se que os indivíduos que possuem dor musculoesquelética apresentam tempos de trabalho significativamente maiores ( $p$-valor=0,032) em relação aos que não tem dor. 
Figura 2: Avaliação da satisfação com a imagem corporal de acordo com o teste das figuras de Stunkard.

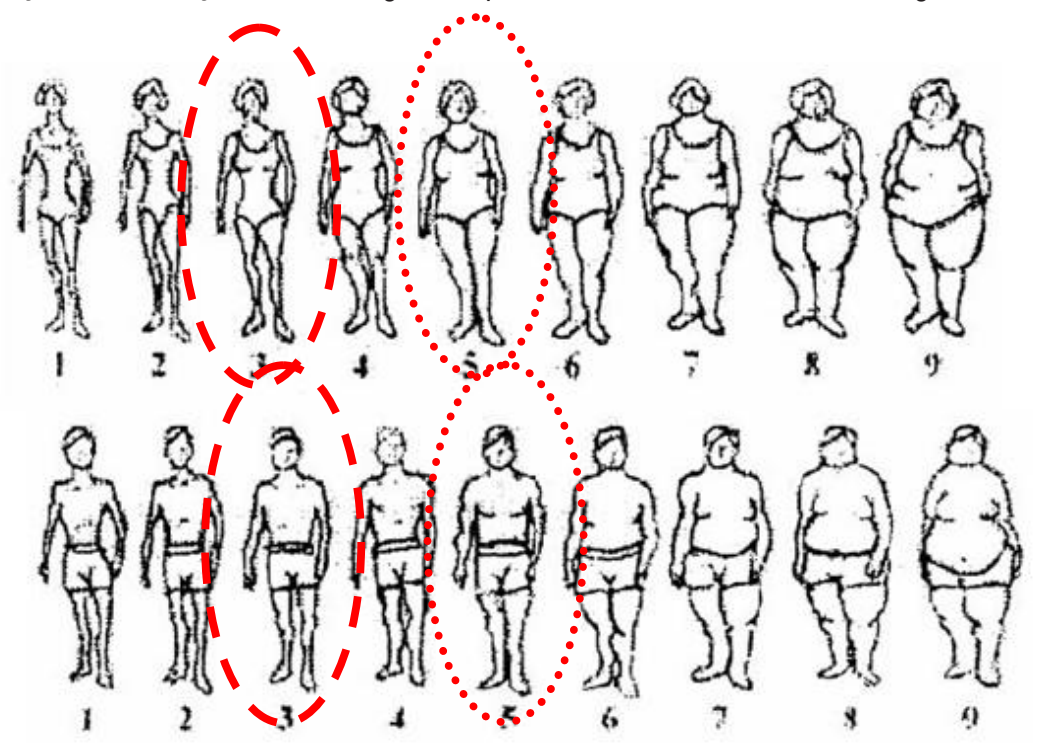

\section{Discussão}

A amostra estudada apresentou características laborais que se reportam à postura sentada. Esta posição possibilita pouca margem de movimentação, tendo como consequência acúmulo de cargas estáticas sobre certos segmentos corporais, predispondo a lesões ocupacionais ${ }^{22,23}$, além disso é sabido que a posição sentada aumenta a pressão intradiscal, elevando assim o risco de hérnia no disco intervertebral24.

O fato da posição dos funcionários exercerem sua atividade laboral ser predominantemente sentada é um dos inúmeros fatores que estão ligados ao aparecimento de dores ${ }^{25}$. Neste estudo 21 referiram sentir algum tipo de dor musculoesquelética, sendo que os pontos mais citados foram coluna cervical, ombros, coluna torácica e coluna lombar.

Estudos apontam que as queixas de dores musculoesqueléticas no trabalho nas regiões de ombro e pescoço estão se tornando tão frequentes quanto as queixas lombares ${ }^{26,27}$. Já outros autores relataram que os trabalhadores administrativos de uma universidade apontaram as regiões da coluna lombar e ombros como os locais mais acometidos ${ }^{28}$.

Em um estudo com operadores de caixas de supermercado, que trabalham predominantemente sentados, observouse que $81 \%$ apresentaram dores na região lombar, $54 \%$ relataram dores nos ombros, $50 \%$ apresentaram dores na coluna dorsal e $49 \%$ no pescoço. Estudos realizados ${ }^{28,29}$ revelam que mais de $70 \%$ das pessoas que trabalham em atividades sedentárias sofrem dores nas regiões lombar e cervical.

Outra informação identificada nos instrumentos de pesquisa foi o cansaço referido pela maioria dos participantes ao final da carga horária de trabalho (35 participantes, 27 referem cansaço); e quando perguntado quanto ao tipo de cansaço ${ }^{16}$ referiu sentir tanto cansaço físico quanto mental. A fadiga promove um conjunto de alterações no organismo resultantes de atividades físicas ou mentais em excesso que levam à sensação de cansaço, podendo reduzir o desempenho laboral e até mesmo causar doenças ocupacionais ${ }^{30}$.

Neste estudo evidenciou-se um ponto benéfico com relação ao desempenho funcional dos voluntários, pois apesar de apresentarem dor e cansaço ao final da jornada de trabalho, a satisfação no trabalho avaliada pelo índice de satisfação no trabalho foi considerada boa. A satisfação no trabalho pode resultar na percepção do indivíduo, sobre até que ponto as atividades que ele desenvolve em seu trabalho, atendem a valores considerados, por ele, como importantes ${ }^{31,32}$;

A satisfação no trabalho está ligada a qualidade de vida em saúde; porém para entender a relação entre satisfação no trabalho e saúde, levou-se em conta que o trabalho é uma das mais importantes maneiras do homem se posicionar como indivíduo; é algo que complementa e dá sentido à vida ${ }^{33,34}$. Por isso o trabalho é visto como um dos componentes da felicidade humana, na qual a felicidade no trabalho é tida como resultante da satisfação de necessidades psicossociais, do sentimento de prazer e do sentido de contribuição no exercício da atividade profissional 34,35 .

Um outro fator que pode interferir na qualidade de vida do indivíduo, além da satisfação laboral é a satisfação corporal, que esta pesquisa apontou que tanto a população masculina quanto a feminina não estão satisfeitos com sua massa 
corporal e gostariam de diminuí-la. Isso fica comprovado quando solicitado o teste de figuras de Stunkard, onde também a maioria dos entrevistados tem como imagem corporal uma figura mais volumosa do que a que realmente gostariam de ser (imagem real mais gorda que imagem ideal). A percepção da imagem corporal e os sentimentos de insatisfação podem influenciar negativamente a vida geral das pessoas, seu desempenho profissional e mesmo o relacionamento interpessoal ${ }^{36}$.

O processo de formação da imagem corporal pode ser influenciado pelo sexo, idade, meios de comunicação, bem como pela relação do corpo com os processos cognitivos como crença, valores e atitudes inseridos em uma cultura ${ }^{34}$,bem como estados emocionais, conflitos psíquicos e interação com os outros seres ${ }^{36}$ e geralmente a insatisfação com o próprio corpo, ou melhor, com a imagem que se tem dele, talvez seja um dos motivos principais que levem as pessoas a iniciar um programa de atividade física ${ }^{37,38,39}$. Este dado corrobora com a presente pesquisa, já que 18 deles não realizam atividade física regular.

A vida sedentária é reconhecida, mais fortemente, como sendo importante fator contribuinte na ausência de saúde e morte precoce. A Organização Mundial da Saúde e a Federação Internacional do Esporte estimam que metade da população mundial seja inativa fisicamente. No Brasil, cerca de $60 \%$ dos brasileiros não praticam nenhum tipo de atividade física ${ }^{40}$.

É sabido que a inatividade física estaria relacionada direta ou indiretamente com dores na coluna. A maior parte da atenção dirige-se a considerá-la um subproduto da combinação da aptidão musculoesquelética deficiente e uma ocupação que force alguma região corporal predisponente à lesão ocupacional ${ }^{41,42}$.

Todos estes fatores combinados podem vir a interferir na qualidade de vida do indivíduo; apesar de neste estudo a qualidade de vida ter sido considerada boa, alguns domínios parecem mais prejudicados que outros sendo eles a dor, podendo ser ocasionada pela posição de trabalho e pelo cansaço físico ao final do dia, à saúde mental, devido à presença de dor e do cansaço mental durante a prática de atividades ocupacionais, e os aspectos gerais de saúde, além de todos os quesitos supracitados. Outro fator que pode ter influenciado é o sedentarismo como argumentado no parágrafo anterior.

A boa qualidade de vida pode estar relacionada ao fato de o trabalho se restringir somente aos turnos trabalhados, ou seja, os funcionários não acumulam atividades extras fora do horário de trabalho, apresentando maior tempo para seu lazer. Em estudo realizado, relacionando horas de trabalho com qualidade de vida pode-se concluir que uma jornada de trabalho saudável pode propiciar melhor ajustamento entre vida profissional e particular, oferecendo tempo para cuidados com a saúde, família e outros assuntos ${ }^{43}$. Os empregados conseguem dedicar-se um período do dia ao trabalho, sem a necessidade de se preocupar com assuntos pessoais. Isso pode repercutir positivamente sobre a qualidade de vida no trabalho, acompanhada da melhoria da produtividade ${ }^{44}$. Sugere-se também que a qualidade de vida no trabalho está diretamente associada à satisfação dos trabalhadores no desempenho de suas funções ${ }^{45}$.

\section{Considerações Finais}

Através deste estudo foi possível observar que a qualidade de vida dos funcionários técnico administrativos da UFSM foi considerada boa, bem como a satisfação no trabalho, porem o estudo demonstrou que os indivíduos apresentaram insatisfação com relação à imagem corporal.

Como a cartilha que foi entregue a cada funcionários participante continha os resultados do estudo, almeja-se que estas informações promovam autoconhecimento por parte dos voluntários e seja motivador, se necessário, para mudanças de atitudes e de comportamentos no próprio estilo de vida.

\section{Referências Bibliográficas}

1. Carvalho VR. Gestão da qualidade: tópicos avançados. São Paulo: Pioneira Thomson Learning; 2004.

2. Michael M. Stress: sinais e causas. São Paulo: Roche; 1998.

3. Coury HJC. Perspectivas e Requisitos para a Atuação Preventiva da Fisioterapia nas Lesões Músculo Esqueléticas. Fisiot Mov 1992-1993; 5(2):63-8.

4. Pirocca, E. Prevalência de Sintomas osteomusculares em trabalhadores da área administrativa da Prefeitura Municipal de Tuparendi-RS. Rio Grande do Sul: Unijui.Trabalho de Conclusão de Curso, 2011. Disponível em: http://bibliodigital. unijui.edu.br:8080/xmlui/handle/123456789/515. Acesso em 27 de novembro de 2014.

5. Grandjean E. Manual de ergonomia: adaptando o trabalho ao homem. Porto Alegre: Artes Médicas; 1998. 
6. VIUDES, P.L.R. LER E DORT no trabalho. Revista eficaz.Revista científica online. Maringá-PR, 2010. Disponível em:<http://www.institutoeficaz.com.br/revistacientifica/wpcontent/uploads/2009/12/Patr\%C3\%ADcia-Lessa-Viudes.pdf>. Acesso em: 13 de novembro 2014.

7. Rabacow FM. Hábitos de Lazer e Índice de Capacidade para o Trabalho em Funcionários de uma Empresa de Produção de Energia. Dissertação de mestrado apresentada para o Programa de Pós-Graduação em Educação Física da Universidade Federal de Santa Catarina na Sub-Área da Atividade Física Relacionada à Saúde; 2008.

8. Marques, Amanda et al. A Ergonomia como um Fator Determinante no Bom Andamento da Produção: um Estudo de Caso. Revista Anagrama: Revista Científica Interdisciplinar da Graduação, São Paulo-SP, ano 4 - edição 1, setembro - novembro de 2010. Disponível em:<http://www.revistas.univerciencia.org/index.php/anagrama/article/ viewDownloadlnterstitial/7143/6535>. Acesso em:10 de novembro de 2014.

9. Dantas EHM. Flexibilidade, alongamento e flexionamento. 4.ed. Rio de Janeiro: Shape; 1999.

10. Endicott J, Nee J, Harrison W, Blumenthal R. Quality of life enjoyment and satisfaction questionnaire: a new easure. Psychopharmacol Bulletin, 29, 1993.

11. Vasconcelos AF. Qualidade de vida no trabalho: origem, evolução e perspectivas. Cadernos de Pesquisa em Administração, v.8, n.1, 2001.

12. Moura, L.L.F. Qualidade de Vida no Trabalho: uma aplicação prática do modelo de Walton no contexto de uma empresa em Picos-PI. 2011. 59p. Monografia (Graduação em Bacharelado em Administração) - Universidade Federal do Piauí, Picos-PI .2011.

13. Deliberato PCP. Fisioterapia Preventiva. São Paulo: Manole; 2002.

14. Barbosa, L. G. Fisioterapia Preventiva nos Distúrbios osteomusculares Relacionados ao Trabalho - DORTs: Fisioterapia do Trabalho Aplicado. 2.ed. Rio Janeiro: Guanabara Koogan; 2009.

15. MARCONI. M. A.LAKATOS. E. M. Fundamentos de Metodologia Científica. 7. Ed. São Paulo: Atlas; 2010.

16. Andrade, M.M de. Introdução à metodologia do trabalho científico: elaboração de trabalhos na graduação. 10. ed. São Paulo: Atlas; 2010.

17. Queiroga MR. Testes e medidas para avaliação da aptidão física relacionada à saúde em adultos. Rio de Janeiro: Guanabara Koogan; 2005.

18. Pimenta CAM. Escalas de avaliação de dor. In: Teixeira MD (ed.) Dor conceitos gerais. São Paulo: Limay 1994; 46-56.

19. Ware JE et al. SF-36 Health Survey: Manual and Interpretation Guide. Boston, The Health Institute, New England Medical Center; 1993.

20. Swan JA, Moraes LFR, Cooper CL. Developing the occupational stress indicator (OSI) for use in Brazil: A report on the reliability and validity of the translated OSI. Stress Medicine1993:9, 247-253.

21. Stunkard AJ, Sorenson T, Schlusinger F. Use of the Danish Adoption Register for the study of obesity and thinness. In S.S. Kety,L.P. Rowland, R.L. Sidman, \& S.W. Matthysse (Eds.) The genetics of neurological and psychiatric. New York: Raven Press; 1983.

22. Marras W. Biomechanics of the human body. In: Salvendy, Handbook of human factors and Ergonomics , 2 ed. Nova lorque: John Wiley \& Sons, 1997.

23. Heuch I et al. The impact of body mass índex on the prevalence of low back pain: the HUNT study. Spine 2010;35(7):764-768.

24. Brito PM et al. Análise da relação entre a postura de trabalho e a incidência de dores na coluna vertebral. Disponível em: http://www.abepro.org.br/biblioteca/enegep2003_tr0406_1582.pdf Data de acesso: 10 de novembro de 2014.

25. Rumaquella M. R.; Menezes, M. S.; Paschoarelli, L. C. ; Santos filho, A.G. "Os efeitos da posição sentada prolongada na coluna vertebral: uma revisão". In: Anais do $8^{\circ}$ congresso Brasileiro de pesquisa e desenvolvimento em design. São Paulo: UNESP, 2008.

26. Westgaard RH, Winkel J. Ergonomic intervention research for improved musculoskeletal health: a critical review. International Journal of Industrial Ergonomics 1997; 20(6):463-500.

27. Vernon H Humphreys BK. Chronic Mechanical Neck Pain in Adults Treated by Manual Therapy: A Systematic Review of Change Scores in Randomized Controlled Trials of a Single Session. The journal of manual \& manipulative therapy, 2009: 16 (2): 42-52.

28. Silva $C D$, Juvêncio JF. Fatores humanos gerais e a aptidão física relacionada à saúde como agentes potencializadores de LER/DORT em trabalhadores de escritório. Rev Min Educ Fís. 2005;13(1):111-29.

29. Batiz EC, Santos AF, Licea OEA. A postura no trabalho dos operadores de checkout de supermercados: uma necessidade constante de análises. Prod. 2009; 19(1):190-201. 
30. Barreto AC, Branco AB. Influência da Atividade Física Sistematizada no Estresse e na Fadiga dos Trabalhadores do Restaurante Universitário da Universidade de Brasília. Revista Brasileira de Atividade Física \& Saúde 2000:5(2)

31. Cardoso, B.S. Prevalência de Distúrbios Osteomusculares em Bancários da Região de Campos Novos. 2012. Disponível em: <http://www.fisioweb.com.br/portal/artigos/categorias/47-art-reumatologia/1325-prevalenciade-disturbiososteomusculares-em-bancarios-da-regiao-de-campos-novos.html>.Acesso em: 27 de novembro de 2014.

32. Coda R. Satisfação no trabalho e políticas de RH:uma pesquisa junto a executivos. In: Bergamini CW, Coda R, orgs. Psicodinâmica da vida organizacional: motivação e liderança. São Paulo: Pioneira, 1990. Cap.4, p.65-85.

33. Alberto LCFR. Os determinantes da felicidade no trabalho: um estudo sobre a diversidade nas trajetórias profissionais de engenheiros [dissertação de mestrado]. São Paulo: Instituto de Psicologia da USP; 2000.

34. Lunnardi Filho. Prazer e sofrimento no trabalho:contribuições à organização do processo do trabalhoda enfermagem. Rev Bras Enferm 1997;50(1):77-92.

35. Robbins, S. P.; Judge, T. A.; Sobral, F. Comportamento organizacional: teoria e pratica no contexto brasileiro. 14. ed. São Paulo: Pearson, 2010.

36. Provencher V. et al. Defined weight expectations in overweight women: anthropometrical, psychological and eating behavioral correlates. Int J Obes.;31(11):1731-38;2007.

37. Volkwein K, MCconatha JT. Cultural contours of the body - The impact of age and fitness. In: Lidor R, Bar-Eli M, editors. Innovations in sport psychology: Linking theory and practice. Tel-Aviv: ISSP, 1997;744-6.

38. Freitas GG. O esquema corporal, a imagem corporal, a consciência corporal e a corporeidade. ljuí: Unijuí. 2004.

39. Fox KR, Corbin CB. The physical self-perception profile: development and Preliminary validation. Journal Sport Exercise Psychological 1989;11:408.

40. Folha de S. Paulo. Datafolha: 60\% dos brasileiros estão parados. Especial: Mexa-se. São Paulo: p. 12, 27/11/1997.

41. Nieman DC. Exercício e saúde. São Paulo: Manole, 1999.

42. Santos AC. O exercício físico e o controle da dor na coluna vertebral. Rio de Janeiro: Medsi; 1996.

43. Carneiro TL, Ferreira MC. Redução de jornada melhora a qualidade de vida no trabalho? A experiência de uma organização pública brasileira. RPOT.2007;7(1):131-158.

44. ALVES, E. F. Programas e Ações em Qualidade de Vida no Trabalho - Revista INTERFACEHS - v.6, n.1, p 63-64. Abril. 2011. Disponível em <http://www.revistas.sp.senac.br/index.php/ITF/article/viewFile/168/180> Acesso em 07 de novembro de 2014.

45. Prati C, Krumenauer R, Blaya C, Bonamigo DR, Boneti C, Lopes MLL. Frequência de LER./DORT: lesões por esforços repetitivos/distúrbios osteomusculares relacionados ao trabalho em digitadores de um jornal em Porto Alegre. Rev Pesq Med. 1999; 33(1-2):34-8.

\section{Ana Paula Ziegler Vey}

Endereço para correspondência - Rua: Fernando Ferrari, n 50, Bairro: Centro, CEP: 97400000 ,

Cidade:Santa Maria, RS, Brasil

E-mail: aninhaziegler@hotmail.com

Lattes: http://lattes.cnpq.br/5413451804582936

Luciane Sanchotene Etchepare Daronco - luetchepare@gmail.com

Andressa Ferreira da Silva - andressaferreiradasilva@hotmail.com

Leonardo Fernandes de Souza - leo.edfisica@hotmail.com

Melissa Medeiros Braz - melissabraz@hotmail.com

Haury Temp - haurytemp@yahoo.com.br

Nadyne Nadyne Rubin - nadynerubin@yahoo.com.br

Tábada Samantha Marques Rosa - sa_marqs@hotmail.com

\section{Enviado em 03 de abril de 2014. Aceito em 15 de dezembro de 2014.}

